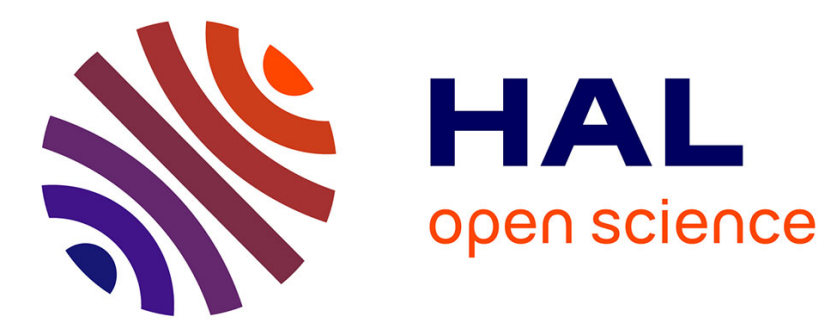

\title{
Immunovirologic control 24 months after interruption of antiretroviral therapy initiated close to HIV seroconversion
}

Laurent Hocqueloux, Asier Saez-Cirion, Christine Rouzioux

\section{- To cite this version:}

Laurent Hocqueloux, Asier Saez-Cirion, Christine Rouzioux. Immunovirologic control 24 months after interruption of antiretroviral therapy initiated close to HIV seroconversion. JAMA Internal Medicine, 2013, 173 (6), pp.475-476. 10.1001/jamainternmed.2013.2176 . pasteur-01420539

HAL Id: pasteur-01420539

https://hal-pasteur.archives-ouvertes.fr/pasteur-01420539

Submitted on 20 Dec 2016

HAL is a multi-disciplinary open access archive for the deposit and dissemination of scientific research documents, whether they are published or not. The documents may come from teaching and research institutions in France or abroad, or from public or private research centers.
L'archive ouverte pluridisciplinaire HAL, est destinée au dépôt et à la diffusion de documents scientifiques de niveau recherche, publiés ou non, émanant des établissements d'enseignement et de recherche français ou étrangers, des laboratoires publics ou privés. 
The authors provide us with a clear message that to improve patients' QoL at the EOL we need to decrease the aggressiveness of care and provide better support to our patients. Hence, urgent change in our practice for patients coming close to the EOL is required. Oncologists need to decrease likely ineffective and toxic chemotherapy, given that this may have a negative impact on both QoL ${ }^{1}$ and survival. ${ }^{3}$ However difficult, we need to have honest EOL discussions with our patients, which can facilitate this transition from disease-directed therapy to care focusing on palliation of symptoms and improving QoL. Previous work from the CWC study has shown that EOL discussions reduce aggressive care and thus improve QoL, result in EOL care more consistent with patients' preferences, ${ }^{4}$ and reduce health care costs in the final week of life. Furthermore, improving patients' symptoms and QoL can be achieved by further training of oncologists in palliative care ${ }^{2}$ by involving other members of the multidisciplinary team (eg, psychologists, chaplains) and by referring patients with advanced, incurable cancer to palliative care. ${ }^{5}$

\section{Haris Charalambous, BM(Soton), MRCP(UK), FRCR(UK)}

Author Affiliation: Consultant Clinical Oncologist, Bank of Cyprus Oncology Centre, Nicosia, Cyprus.

Correspondence: Dr Charalambous, Consultant Clinical Oncologist, Bank of Cyprus Oncology Centre, 32 Acropoleos Ave, 2006 Strovolos, Nicosia, Cyprus (haris .charalambous@bococ.org.cy).

Conflict of Interest Disclosures: None reported.

1. Zhang B, Nilsson ME, Prigerson HG. Factors important to patients' quality of life at the end of life. Arch Intern Med. 2012;172(15):1133-1142.

2. Peppercorn JM, Smith TJ, Helft PR, et al. American Society of Clinical Oncology statement: toward individualized care for patients with advanced cancer. J Clin Oncol. 2011;29(6):755-760.

3. Greer JA, Pirl WF, Jackson VA, et al. Effect of early palliative care on chemotherapy use and end-of-life care in patients with metastatic non-small-cell lung cancer. J Clin Oncol. 2012;30(4):394-400.

4. Mack JW, Weeks JC, Wright AA, Block SD, Prigerson HG. End-of-life discussions, goal attainment, and distress at the end of life: predictors and outcomes of receipt of care consistent with preferences. J Clin Oncol. 2010;28 (7):1203-1208

5. Smith TJ, Temin S, Alesi ER, et al. American Society of Clinical Oncology Provisional Clinical Opinion: the integration of Palliative Care into Standard Oncology Care. J Clin Oncol. 2012;30(8):880-887.

\section{In reply}

We agree with Dr Charalambous that the message of our findings is that to improve the quality of life for patients with advanced cancer at the end of life, there needs to be a reduction in the intensity of medical care (eg, the number of intensive care unit stays, use of ventilators) they receive, as well as heightened attention to their psychosocial and spiritual needs. Dr Charalambous has taken these results as evidence to support his recommendation that oncologists be trained in palliative care and psychologists, chaplains, and palliative care clinicians be involved in multidisciplinary care of the patient. This suggestion appears to be consistent with our findings, but there is an additional related "takehome" point.

Many oncologists express frustration and a sense of futility in caring for their incurable patients. Oncologists have shared that they sometimes feel that they have little to offer such patients. This may prompt a need to respond by offering overly aggressive medical care. Many oncologists have voiced a preference for others whom they consider better trained in these areas to attend to advanced cancer patients' psychosocial needs. Patients and family members, however, express fears and disappointment regarding what they perceive to be oncologist emotional detachment and abandonment. What the results of our report indicate is that building a therapeutic alliance with patients with advanced cancer (eg, a sense of trust, empathic concern, shared goals) may be the best and most potent medicine oncologists have to offer their dying patients. Oncologists who are able to be emotionally available and attentive to patients' psychosocial and spiritual needs have the power to improve the quality of life of their patients with advanced cancer who are confronting death.

Holly G. Prigerson, PhD

Baohui Zhang, MS

Matthew E. Nilsson, BS

Author Affiliations: Center for Psychosocial Epidemiology and Outcomes Research (Dr Prigerson, Ms Zhang, and Mr Nilsson) and Division of Population Sciences, Department of Medical Oncology (Dr Prigerson), Dana-Farber Cancer Institute, and Department of Psychiatry, Brigham and Women's Hospital, Harvard Medical School (Dr Prigerson), Boston, Massachusetts.

Correspondence: Dr Prigerson, The Dana Farber Cancer Institute, Center for Psychosocial Epidemiology and Outcomes Research, 1134 Dana Bldg, 450 Brookline Ave, Boston, MA 02115 (Holly_Prigerson@dfci.harvard.edu).

Conflict of Interest Disclosures: None reported.

\section{Immunovirologic Control 24 Months After Interruption of Antiretroviral Therapy Initiated Close to HIV Seroconversion}

\section{L}

odi et al ${ }^{1}$ recently reported that 11 of 259 patients (4.2\%) starting combined antiretroviral therapy (cART) at the time of primary human immunodeficiency virus type 1 (HIV-1) infection (PHI) still controlled HIV replication $(<50$ copies $/ \mathrm{mL}) 24$ months after treatment interruption. They pointed out that the proportion of these "posttreatment controllers" (PTCs) was substantially lower than the $15.6 \%$ we reported, ${ }^{2}$ and speculated that this difference might have been because we excluded patients who restarted therapy less than 24 months after treatment interruption and used newer and more powerful drugs. However, the period of treatment initiation in our study was similar to that reported by Lodi et $\mathrm{al}^{1}$ (19962007 and 1996-2009, respectively), and the drug regimens we used were part of the contemporary standard-ofcare recommendations. In addition, $91 \%$ of our patients had highly symptomatic PHI and, thus, a poor prognosis. Finally, we did not exclude patients who resumed therapy less than 24 months after treatment interruption but included them in the "noncontrollers" group, which resulted in a lower frequency of posttreatment controllers.

Rather than inclusion biases, we believe that the high frequency of PTCs observed in our study may have been 
due to the long duration of cART before treatment interruption. To our knowledge, only 4 cohort studies have described patients fulfilling the criteria for PTC, and all included patients who started treatment during PHI. It is particularly noteworthy that the frequency of PTCs in these cohort studies increased linearly with the median duration of cART prior to treatment interruption, from $4.2 \%$ in the CASCADE (Concerted Action on Seroconversion to AIDS and Death in Europe) study ${ }^{1}$ (median duration of cART, 1.3 years) to $7.9 \%$ in the ANRS (Agence Nationale de Recherche sur le Sida) CO6 PRIMO cohort $^{3}$ (median, 1.4 years), $8.3 \%$ in a Dutch cohort $^{4}$ (median, 1.7 years), and $15.6 \%$ in our study ${ }^{2}$ (median, 2.4 years). In the SPARTAC (Short Pulse Anti Retroviral Therapy at HIV Seroconversion) study, ${ }^{5}$ in which the median duration of cART was 0.25 years, there were no PTCs. Note that no more than $0.5 \%$ of patients spontaneously control HIV replication after PHI.

We believe the main reason why PTC status is a rare phenomenon is the small number of patients who are prescribed long-term treatment at the time of PHI and then undergo scheduled treatment interruption (only 1\% of patients in the CASCADE study).

At all events, evidence supporting the existence of PTC status continues to accumulate. This phenomenon has important implications in the search for a functional cure for HIV infection and further supports early treatment, especially given the potency and good tolerability of current drugs.

\section{Laurent Hocqueloux, MD \\ Asier Saez-Cirion, PhD \\ Christine Rouzioux, PharmD, PhD}

Author Affiliations: Services des Maladies Infectieuses, Centre Hospitalier Regional d'Orleans, La Source, Orleans, France (Dr Hocqueloux); Institut Pasteur, Unite de Regulation des Infections Retrovirales, Paris, France (Dr Saez-Cirion); Assistance Publique-Hôpitaux de Paris, Centre Hospitalier Universitaire (CHU) NeckerEnfants Malades, Laboratoire de Virologie, Paris (Dr Rouzioux); and Infections à Vih, Réservoirs, Pharmacologie des Antirétroviraux et Prévention de la Transmission Mère Enfant (EA 3620), Universite ParisDescartes, Sorbonne Paris Cite, Paris (Dr Rouzioux). Correspondence: Dr Hocqueloux, Service des Maladies Infectieuses et Tropicales, Centre Hospitalier Regional d'Orleans, La Source, BP 86709, 45067 Orleans CEDEX 2, France (laurent.hocqueloux@chr-orleans.fr).

Conflict of Interest Disclosures: None reported.

1. Lodi S, Meyer L, Kelleher AD, et al. Immunovirologic control 24 months after interruption of antiretroviral therapy initiated close to HIV seroconversion. Arch Intern Med. 2012;172(16):1252-1255.

2. Hocqueloux L, Prazuck T, Avettand-Fenoel V, et al. Long-term immunovirologic control following antiretroviral therapy interruption in patients treated at the time of primary HIV-1 infection. AIDS. 2010;24(10):1598-1601.

3. Goujard C, Girault I, Rouzioux C, et al; ANRS CO6 PRIMO Study Group. HIV-1 control after transient antiretroviral treatment initiated in primary infection: role of patient characteristics and effect of therapy. Antivir Ther. 2012 17(6):1001-1009.

4. Steingrover R, Pogány K, Fernandez Garcia E, et al. HIV-1 viral rebound dynamics after a single treatment interruption depends on time of initiation of highly active antiretroviral therapy. AIDS. 2008;22(13):1583-1588.

5. Hamlyn E, Ewings FM, Porter K, et al; INSIGHT SMART and SPARTAC Investigators. Plasma HIV viral rebound following protocol-indicated cessation of ART commenced in primary and chronic HIV infection. PLoS One. 2012; 7(8):e43754

\section{In reply}

Hocqueloux et al propose that longer duration of combined antiretroviral therapy (cART) in primary human immunodeficiency virus (HIV) infection (PHI) is associated with a higher probability of creating posttreatment controllers (PTCs). They cite as evidence the superior proportion of PTCs in their study compared with ours (15.6\% vs 5.5\%), as well as a number of studies that would support their hypothesis. ${ }^{1-5}$ While longer duration of cART administered during PHI may lead to a higher proportion of individuals controlling viral replication on its withdrawal, a hypothesis not supported by their own study or the ANRS (Agence Nationale de Recherche sur le Sida) CO6 PRIMO study by Goujard et al, ${ }^{3}$ studies differ by a number of factors other than treatment duration. Therefore, inference about the effect of cART duration from raw figures derived from published studies is inappropriate. In any case, it is erroneous to state that there were no PTCs in the SPARTAC (Short Pulse Anti Retroviral Therapy at HIV Seroconversion) study, which could not be deduced from the citation provided. ${ }^{5}$

In the study by Hocqueloux et al, ${ }^{1}$ the time at risk following treatment discontinuation was conditioned on not resuming cART within 24 months of discontinuation. In our study no such condition was required, since all individuals who had interrupted cART (and were suppressed at the time) contributed to the risk set until they experienced an event (viral rebound) or their follow-up was censored. The comparative figure from our study would be $8.5 \%$ (11 of 130 who did not reinitiate cART within 24 months of discontinuation).

Finally, the denominator (25 629) used to derive the 1\% from CASCADE (Concerted Action on Seroconversion to AIDS and Death in Europe) who were treated at the time of PHI and subsequently stopped treatment is inaccurate and results in underestimating that proportion for our study. The appropriate denominator is of individuals with an HIV test interval less than 3 months, with at least 3 months of followup, at least 1 HIV RNA measurement, and who seroconverterted from 1996 onwards and would have, therefore, had the opportunity to have been treated with cART. Of 4010 such individuals in CASCADE, 259 (6.5\%) interrupted cART.

In conclusion, while the original study by Hocqueloux et al highlighted the existence of PTC, the key question is whether early treatment during PHI can help establish posttreatment control.

$$
\begin{aligned}
& \text { Kholoud Porter, PhD } \\
& \text { Sara Lodi, PhD, MSc } \\
& \text { Laurence Meyer, MD, PhD }
\end{aligned}
$$

Author Affiliations: Medical Research Council, Clinical Trials Unit, London, United Kingdom (Dr Porter); Instituto de Salud Carlos III, Centro Nacional de Epidemiologia, Madrid, Spain (Dr Lodi); and University Paris Sud, INSERM CESP U1018, Assistance PubliqueHôpitaux de Paris, le Kremlin Bicetre, Paris, France (Dr Meyer).

Correspondence: Dr Porter, MRC Clinical Trials Unit, Aviation House, 125 Kingsway, London WC2B 6NH, United Kingdom (kp@ctu.mrc.ac.uk).

Conflict of Interest Disclosures: Dr Porter has received an honorarium from Tibotec. 
1. Hocqueloux L, Prazuck T, Avettand-Fenoel V, et al. Long-term immunovirologic control following antiretroviral therapy interruption in patients treated at the time of primary HIV-1 infection. AIDS. 2010;24(10):1598-1601.

2. Lodi S, Meyer L, Kelleher AD, et al. Immunovirologic control 24 months after interruption of antiretroviral therapy initiated close to HIV seroconversion. Arch Intern Med. 2012;172(16):1252-1255.

3. Goujard C, Girault I, Rouzioux C, et al; ANRS CO6 PRIMO Study Group. HIV-1 control after transient antiretroviral treatment initiated in primary infection: role of patient characteristics and effect of therapy. Antivir Ther. 2012; 17(6):1001-1009.

4. Steingrover R, Pogány K, Fernandez Garcia E, et al. HIV-1 viral rebound dynamics after a single treatment interruption depends on time of initiation of highly active antiretroviral therapy. AIDS. 2008;22(13):1583-1588.

5. Hamlyn E, Ewings FM, Porter K, et al; INSIGHT SMART and SPARTAC Investigators. Plasma HIV viral rebound following protocol-indicated cessation of ART commenced in primary and chronic HIV infection. PLoS One. 2012; 7(8):e43754.

\section{High-Five for High-Sensitivity Cardiac Troponin T: Depends on the Precision and Analytical Platform}

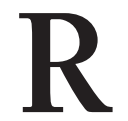
eichlin and colleagues ${ }^{1}$ have developed an algorithm for ruling-in or ruling-out myocardial infarction (MI) within 1 hour after presentation using high-sensitivity cardiac troponin $\mathrm{T}$ (hs-cTnT) for patients within the Advantageous Predictors of Acute Coronary Syndrome Evaluation study. The data analyses using classification and regression tree analysis for ruling-in via a training and validation set are powerful for supporting a 1-hour sampling/testing algorithm, albeit there is a major limitation in that both groups are from the same study population. ${ }^{2}$ Most strikingly, however, is the analytical criteria that was developed in this study to rule-in an MI, specifically, that either a hs-cTnT concentration of $52 \mathrm{ng} / \mathrm{L}$ or greater (to convert to micrograms per liter, multiply by 0.001 ) or a change (delta) in hs-cTnT concentrations of $5 \mathrm{ng} / \mathrm{L}$ or greater within 1 hour was used to rule-in. ${ }^{1}$ The absolute concentration of $52 \mathrm{ng} / \mathrm{L}$ is not the published 99th percentile for this assay ${ }^{3}$ and is in fact similar to the fourth generation's troponin T 10\% coefficient of variation (CV) concentration of $0.03 \mathrm{ng} / \mathrm{L}$, which has been shown in a large prospective study to predict death within 30 days. ${ }^{4}$ More intriguing is the 5-ng/L absolute change in hs-cTnT concentrations. On the basis of reported precision data, the $10 \%$ $\mathrm{CV}$ for the hs-cTnT is $13 \mathrm{ng} / \mathrm{L}^{1}$; using established laboratory medicine practices, acceptable variation would permit concentrations ranging from $15.6 \mathrm{ng} / \mathrm{L}$ to $10.4 \mathrm{ng} / \mathrm{L}$ around this point estimate. Thus, the imprecision of the assay alone could result in a change of $5 \mathrm{ng} / \mathrm{L}$ or greater in absence of any clinical change.

To avoid this assay imprecision trap, one may wish to (1) incorporate a longer time between samples to rule-in (ie, 3 hours; as was recently suggested for high-sensitivity assays $)^{5}$ or (2) use a more precise platform to measure hscTnT. ${ }^{3}$ For the first point, the longer the time between blood sampling, the more likely that the troponin concentration will increase with the difference (delta) exceeding the analytical imprecision. For the second point, there are analytical platforms that have different precision profiles. The E-modular platform is more precise than the Elecsys 2010 platform, with the latter often yielding imprecision that would result in $5 \mathrm{ng} / \mathrm{L}$ or greater difference in concentrations. ${ }^{3}$ Finally, prospective studies assessing this algo- rithm should compare this shorter interval (ie, 1 hour) to a longer time interval (ie, 3 hours) for measurements and should also report on the platform and precision data for the hs-cTnT testing during the study. These are important points that should be addressed before considering adopting this algorithm for clinical care.

\section{Peter A. Kavsak, PhD}

Author Affiliation: Department of Pathology and Molecular Medicine, McMaster University, Hamilton, Ontario, Canada

Correspondence: Dr Kavsak, Juravinski Hospital and Cancer Centre, 711 Concession Street Hamilton, ON L8V 1C3, Canada (kavsakp@mcmaster.ca).

Conflict of Interest Disclosures: Dr Kavsak has received grants/consultant/honorariums from Abbott Diagnostics, Beckman Coulter, Randox Laboratories, and Roche Diagnostics. He is listed as an inventor on patents filed by McMaster University related to laboratory testing in acute cardiac care.

1. Reichlin T, Schindler C, Drexler B, et al. One-hour rule-out and rule-in of acute myocardial infarction using high-sensitivity cardiac troponin T. Arch Intern Med. 2012;172(16):1211-1218.

2. Henderson MP, Pond GR, Kavsak PA. Statistical and analytical approaches for assessing biomarkers: new approaches, new technologies, with the sameold rigor for evaluation. Clin Biochem. 2012:45(3):187-188.

3. Saenger AK, Beyrau R, Braun S, et al. Multicenter analytical evaluation of a high-sensitivity troponin T assay. Clin Chim Acta. 2011;412(9-10):748-754.

4. Devereaux PJ, Chan MT, Alonso-Coello P, et al; Vascular Events In Noncardiac Surgery Patients Cohort Evaluation (VISION) Study Investigators. Association between postoperative troponin levels and 30-day mortality among patients undergoing noncardiac surgery. JAMA. 2012;307(21):2295-2304.

5. Thygesen K, Mair J, Giannitsis E, et al; Study Group on Biomarkers in Cardiology of ESC Working Group on Acute Cardiac Care. How to use highsensitivity cardiac troponins in acute cardiac care. Eur Heart J. 2012;33(18) 2252-2257.

\section{Testing Times: We Are Still Some Way From Getting the Best Out of Sensitive Troponin Assays}

$\mathrm{W}$ ewould like to commend Reichlin et al for their novel algorithm for the rule-out and rule-in of acute myocardial infarction using highsensitivity cardiac troponin $T$. This study gives promise to the development of clinically relevant rapid assessment strategies, which will be vital in reducing emergency department (ED) overcrowding, patient inconvenience, and associated health care costs. ${ }^{2}$ However, this study raises some unanswered questions in the development of early diagnostic strategies using sensitive troponin assays.

Recruitment rates in this study seem very low compared with prior published work. ${ }^{3}$ We would suggest that a multicenter study that includes unselected patients with chest pain should recruit far in excess of 1247 patients over a 3-year period. Our own average-size United Kingdom center with an annual ED census of 60000 sees on average 2.6 patients per day who fulfill the inclusion criteria for this algorithm. Even when taking into account a dropout rate of $10 \%$, one would expect each site to recruit more than 800 patients per year. This suggests that the population tested within the study by Reichlin et al ${ }^{1}$ may have been subject to a selection bias and questions the applicability of their results to clinical practice. 